\title{
Physical meaning of pulse signal characteristics in TCM and the latest research progress of its recognition methods
}

\author{
Yuesheng Lou \\ Yiwu Industrial \& Commercial College, Yiwu, Zhejiang, China, 322000
}

Keywords: Pulse signal characteristics in TCM; Physical meanings; Identification method; Fuzzy neural network; BP neural network.

\begin{abstract}
The objective of this study is to clarify the physical meaning and identification method of pulse signal characteristics in traditional Chinese medicine (TCM), realize the quantification of clinical pulse signal, and improve the diagnostic accuracy of TCM pulse condition. In this research, MP150 multipurpose polygraph is used to collect six pulse conditions (chord pulse, deep pulse, smooth pulse, thready pulse, moderate pulse, and flat pulse) of 100 volunteers, describe and analyze the signal characteristic parameters of pulse conditions, and evaluate the correlation between the signal characteristic parameters of pulse conditions and their types. In addition, BP neural network, fuzzy C-means method and fuzzy neural network are adopted to recognize six kinds of pulse signals, and the recognition rate under different methods is calculated. The results show that MP150 multipurpose polygraph can obtain the characteristic parameters of the pulse signal, and the parameters are correlated with the state of the pulse tube. The types of pulse conditions can be distinguished according to the signal characteristics of pulse conditions. The recognition rate of pulse condition by fuzzy neural network is significantly higher than that of BP neural network and fuzzy C-means method $(\mathrm{P}<0.05)$. Therefore, the signal characteristics of different pulse conditions are somewhat different, and the fuzzy neural network can accurately identify the pulse signals, which provides experimental data for the subsequent diagnosis and treatment of TCM.
\end{abstract}

\section{Introduction}

TCM is a comprehensive discipline that studies the transformation rules of health and disease in human life activities as well as prevention, diagnosis, treatment, rehabilitation, and health care [1]. It is the heritage of the Chinese nation. Through the polishing and precipitation of history, it has formed a complete theoretical system and formed four unique diagnostic methods of "look, listen, question, and feel the pulse". The traditional method of diagnosis and examination of the pulse condition is called pulse diagnosis, also known as pulse-taking or palpation, which is the main basis for the diagnosis of Chinese medicine. As the earliest work on pulse condition in China, "The Pulse Classic" marks the gradual formation and improvement of the theory of pulse condition in China [2]. The heart and vein are the main organs that form the pulse. The pulse source is the heart, and the pulse is the specific expression of the heart function. The contraction function of the vessels is an important condition for the circulation of qi and blood. Therefore, the formation of the pulse condition is directly related to the heartbeat of the heart, the ebb and flow of heart qi, the circulation of the veins, and the profit and loss of qi and blood. Therefore, pulse conditions can be used to diagnose diseases [3, 4].

Studies have shown that the pulse condition can reflect the state of the body, and when the body function changes, the pulse condition will also change. Therefore, the nature and location of the disease can be diagnosed clinically according to the pulse condition, and the severity of the disease can also be inferred $[5,6]$. With the continuous improvement and development of the theory of pulse condition, pulse condition has been paid more and more attention and accepted by foreign countries. Traditional diagnosis of pulse condition mainly relies on doctors cutting patients' arterial pulse with their fingers and judging patients' symptoms according to their own experience, which leads to certain deviations in diagnosis and treatment by different doctors and causes psychological distress to 
patients [7]. At present, some scholars combine computer recognition technology with pulse diagnosis to replace the traditional diagnosis method and improve the clinical diagnosis rate. However, pulse characteristics are relatively complex. For the diagnosis of disease, it needs to integrate and analyze all pulse characteristics, which requires computer diagnosis with powerful recognition function [8]. At present, time-domain analysis method is the main method for extracting pulse characteristics, which is relatively simple and easy to analyze pulse signals [9]. In order to more objectively and intelligently describe the correlation between pulse condition and disease, many scholars have done a lot of studies, among which some scholars have shown that wavelet transform detection and modal energy quotient can effectively distinguish normal people from patients with heart disease and patients with hypertension [10]. However, there is a lack of description of the characteristics of pulse signal in TCM, and there is no complete literature to show the research progress of its identification methods. It is impossible to compare and analyze which calculation method is more suitable for pulse signal identification, which limits the clinical application of pulse diagnosis.

To sum up, in order to make the pulse characteristics understood by more people and distinguish the types of pulse conditions according to the pulse signal characteristics, six pulse conditions of 40 volunteers are collected, and the signal differences between different pulse conditions are compared. In addition, the accuracy of three algorithms in pulse recognition is compared, which provides an experimental scheme for clinical pulse recognition.

\section{Methods}

\subsection{Collection of pulse signals}

Forty volunteers are recruited and six pulse conditions (chord pulse, deep pulse, smooth pulse, thready pulse, moderate pulse, and flat pulse) of the 40 volunteers are collected with MP150 multipurpose polygraph. These six are pulse signals that produce higher frequencies in the patient. In order to avoid some adverse external factors, the volunteers are first allowed to rest for about 20 minutes in a quiet state, so that the volunteers' qi and blood are relatively calm. Then the volunteers place their palms on the table, with their hands at the same level as their hearts, so as to ensure that the volunteers' blood and air are flowing smoothly. MP150 multipurpose polygraph is used to collect the pulse of the volunteers' left hand. And the characteristic parameters of different pulse conditions are recorded and the signal parameters of different pulse conditions are obtained: t, h1, h3, h4, h3/h1, $\mathrm{h} 4 / \mathrm{h} 1$.

\subsection{The identification of pulse condition by fuzzy C-Means clustering algorithm}

Fuzzy C-Means clustering algorithm strictly divides each object to be identified into a certain class. $\mathrm{x}(\mathrm{i}=1,2,3 \ldots \mathrm{n})$ is the sample set composed of $\mathrm{n}$ samples, $\mathrm{c}$ is the number of predetermined categories, $\mu \mathrm{j}(\mathrm{xi})(\mathrm{j}=1,2,3 \ldots \mathrm{n})$ is the membership function of the ith sample for the $\mathrm{j}$ class. The cluster loss function defined by the membership function is as follows.

$$
J_{\mathrm{f}}=\sum_{\mathrm{j}=1}^{\mathrm{c}} \sum_{\mathrm{i}=1}^{\mathrm{n}}\left[\mu_{\mathrm{j}}\left(\mathrm{x}_{\mathrm{i}}\right)\right]^{\mathrm{b}}\left\|\mathrm{x}_{\mathrm{i}}-\mathrm{m}_{\mathrm{j}}\right\|^{2}
$$

Among them, $\mathrm{b}>1$, which is a fuzzy degree constant that can control the result of clustering. When the fuzzy C-means algorithm converges, all kinds of clustering centers and membership values of each sample for all kinds are obtained, thus completing the fuzzy clustering differentiation. The classified value is compared with the original value to calculate the recognition accuracy of fuzzy C-means algorithm.

\subsection{The identification of pulse condition by BP neural network}

BP neural network estimates the error of the direct leading layer of the output layer according to the error after the output, and then uses this error to estimate the error of the previous layer, so that the error estimation of other layers can be obtained after the backpropagation of one layer after another. 
Figure 1 is the structure diagram of BP neural network, which is divided into two parts: forward transmission of signals and back propagation of errors. It uses the steepest descent method and continuously adjusts the weights and thresholds of the network through back propagation, so as to minimize the average and minimum errors of the network. In this research, BP neural network is used to select logsig as the transfer function, and the classification is based on the "maximum rule" to compare the obtained value with the original value and calculate the recognition accuracy of BP neural network.

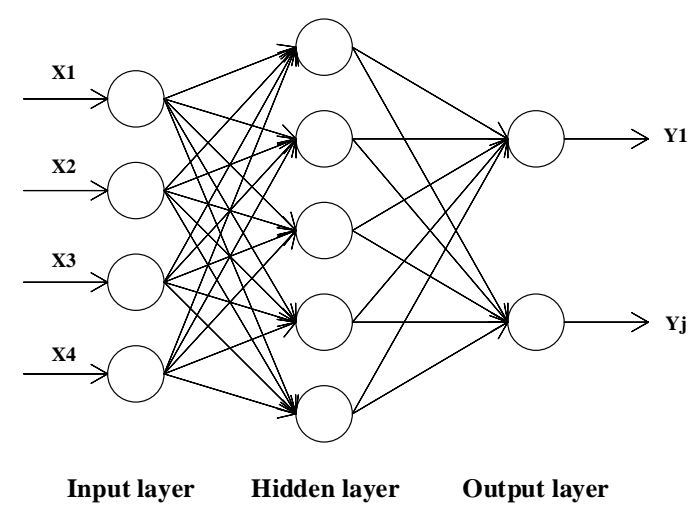

Figure 1. BP neural network structure

\subsection{The identification of pulse condition by fuzzy neural network}

The combination of fuzzy technology and neural network technology constructs a kind of neural network which can process fuzzy information automatically. The network is composed of antecedent network and antecedent network. Antecedent network is composed of the first four layers, which are used to match the antecedents of fuzzy rules. Antecedent network is simplified to the last layer, which is used to generate antecedents of fuzzy rules. Figure 2 shows the structure of fuzzy neural network.

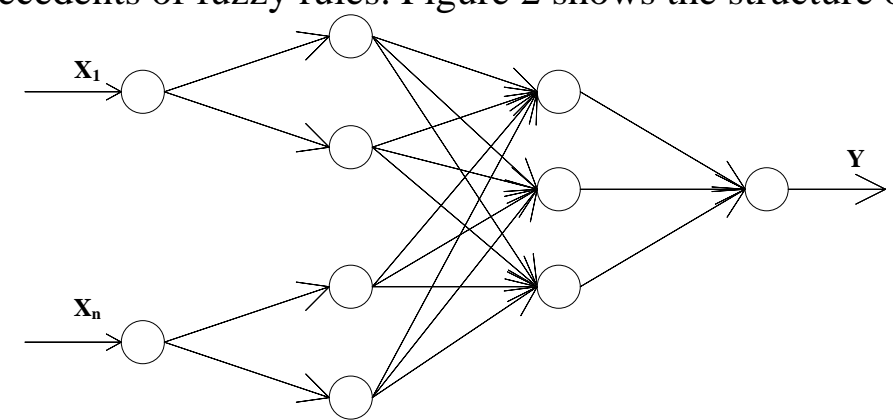

Figure 2. Structure of fuzzy neural network

In this paper, the ANFIS editor is used in the fuzzy neural network, so as to optimize and predict the membership function, obtain the required data, and compare the obtained value with the original value to calculate the recognition accuracy of the fuzzy neural network.

\subsection{Statistical analysis}

SPSS 19.0 software is used for statistical analysis. The measurement data obtained are represented by $\overline{\mathrm{x}} \pm \mathrm{SD}$, and $\mathrm{t}$ test is used for inter-group comparison. The obtained enumeration data are expressed as a percentage (\%) and compared between groups by $\chi^{2}$ test. When $\mathrm{P}<0.05$, it is considered that there is a statistical difference between the comparison groups.

\section{Results}

\subsection{Analysis of characteristic parameters of pulse signal}

MP150 multipurpose polygraph is used to collect six pulse conditions (string pulse, sink pulse, slide pulse, thready pulse, moderate pulse, and flat pulse) of 40 volunteers, and to analyze the 
physical meaning of pulse signal characteristics. Figure 3 shows the characteristic schematic diagram of pulse wave. In the figure, $A$ is the starting point, $B$ is the main wave peak, $C$ is the tidal front valley, $\mathrm{D}$ is the heavy stroke front wave, $\mathrm{E}$ is the descending middle isthmus and $\mathrm{F}$ is the heavy stroke wave. t, h1, h3, and h4 are characteristic parameters of pulse signal. Among them, h1 is the main wave amplitude, which is related to the compliance of the great artery and the ejection ability of the left ventricle. When the compliance of the great artery and the ejection ability of the left ventricle are stronger, h1 is higher. $\mathrm{h} 3$ is the amplitude of the front pulse of the heavy pulse. When the elasticity or peripheral resistance of human arterial blood vessels change, h3 will also change accordingly. When the amplitude of $\mathrm{h} 3$ increases, the phase before improving heavy pulse will advance, which indicates that when the arteries are under high resistance and high tension, the conduction velocity of pulse wave reflection will also accelerate. h4 is the range of descending middle isthmus, which increases with the increase of peripheral arterial resistance, and is also affected by aortic valve closure function. $\mathrm{h} 3 / \mathrm{h} 1$ changes according to the peripheral resistance and compliance of the vascular wall, while h4/h1 increases with the increase of the peripheral resistance of the vascular wall.

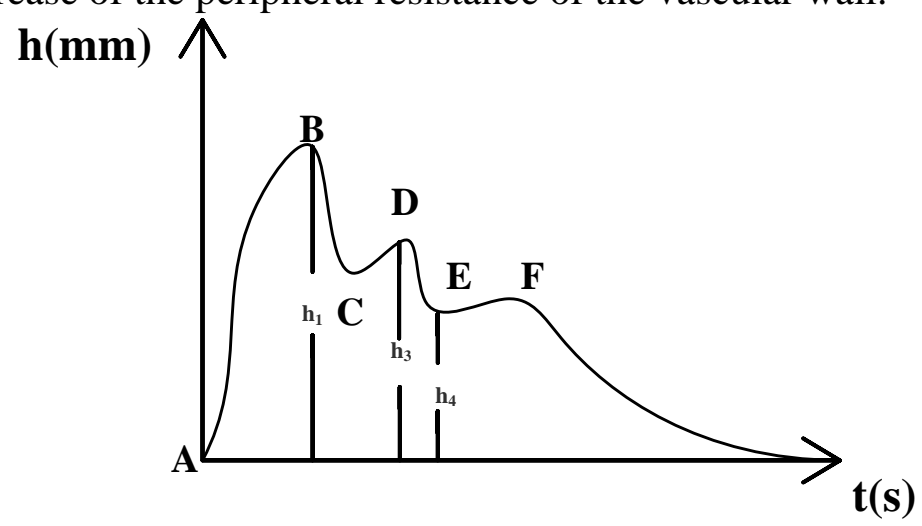

Figure 3. Schematic diagram of the pulse wave

\subsection{Comparison of signal characteristic parameters of six types of pulse conditions}

The pulse parameters of the six obtained pulse conditions are compared and analyzed to explore the correlation between the pulse signal characteristic parameters and the type of pulse conditions. Figure 4 shows the comparison of pulse parameters between different types of pulse conditions. Among them, figure 4A shows the comparison results of height parameters of each time phase of six pulse conditions, and figure $4 \mathrm{~B}$ shows the comparison of pulse cycle of six pulse conditions. It can be observed from figure 4A that thready pulse h4 is almost zero, which can be separated from other pulse conditions according to this index. The $\mathrm{h} 4 / \mathrm{h} 1$ of chord pulse is obviously higher than that of other pulse conditions, and the h4/h1 value of deep pulse is the smallest among the other four pulse conditions except the thready pulse. The flat pulse is a normal pulse, and the parameters of its three peaks decrease in order. It can be observed from figure B that the pulsating period of moderate pulse is higher than that of other pulses. So it can be distinguished from other pulses according to this characteristic.

To sum up, the signal characteristic parameters of each pulse are different and can be distinguished according to their differences, which provides theoretical support for subsequent pulse diagnosis. 


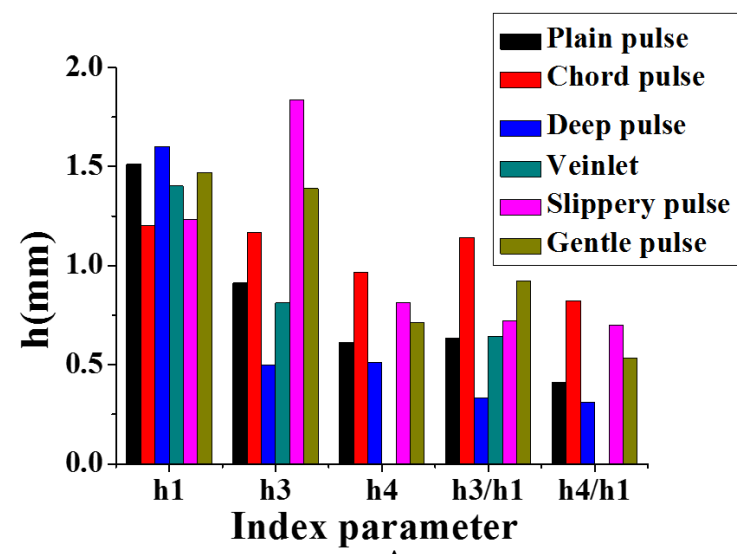

A

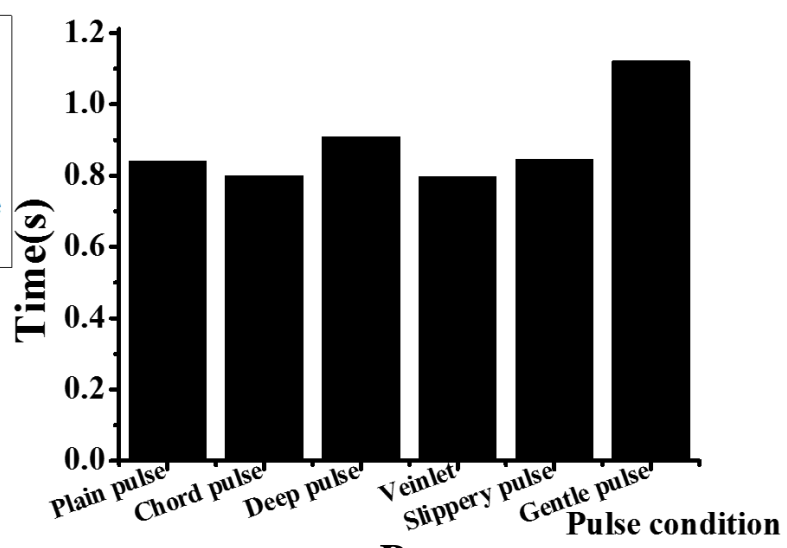

B

Figure 4. Comparison of pulse parameters between different types of pulse conditions Note: figure A shows the comparison of height parameters of each phase of six pulse conditions, and figure $\mathrm{B}$ shows the comparison of pulse cycle of six pulse conditions

\subsection{Comparison of pulse signal classification and recognition}

BP neural network, fuzzy C-means method, and fuzzy neural network are used to identify six kinds of pulse signals, and the obtained data are compared with the original data to calculate the recognition accuracy of each method. Figure 5 shows the comparison of the identification accuracy of the three methods. It can be observed from the figure that the accuracy of the fuzzy neural network in pulse recognition is significantly higher than the BP neural network and the fuzzy C-mean $(\mathrm{P}<0.05)$. Since the fuzzy C-means recognizes the pulse by the cluster center point and membership function, its accuracy will be affected by the cluster center point and the fuzzy function rule, thus reducing its accuracy. BP neural network has good generalization, it has many hidden layers, which makes it have high recognition. The fuzzy neural network combines the neural network with the fuzzy theory. It takes the gaussian function as its membership function, and the weight is adjusted by back propagation, which makes its error smaller and the recognition accuracy increased.

To sum up, the fuzzy neural network has the highest identification rate of pulse condition, which is almost $100 \%$. Applying it to clinical practice can effectively improve the efficiency of treatment, which provides experimental data for clinical pulse condition identification.

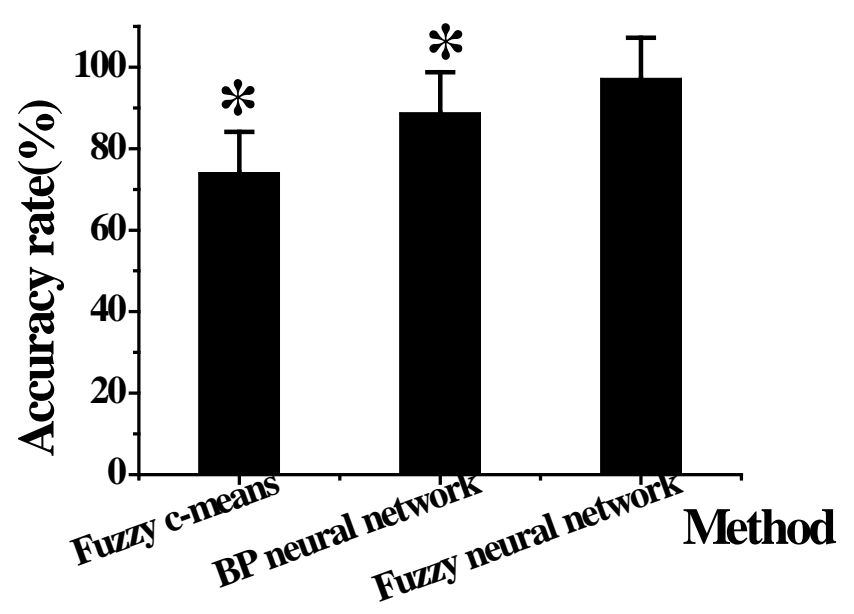

Figure 5. Comparison of recognition accuracy of the three methods

Note: $*$ in the figure indicates that there is a significant difference compared with fuzzy neural network, $\mathrm{P}<0.05$ 


\section{Conclusion}

Pulse condition is an essential factor in TCM diagnosis. In this research, MP150 multipurpose polygraph is used to collect six pulse conditions (chord pulse, deep pulse, smooth pulse, thready pulse, moderate pulse and flat pulse) of 40 volunteers, and the data obtained are accurate and reliable. Meanwhile, the characteristic parameters of the pulse condition are analyzed, and it is found that when the compliance of the great artery and the ejection ability of the left ventricle are stronger, h1 is higher. When the elasticity or peripheral resistance of human arteries change, h3 will change accordingly. h4 increases with the increase of peripheral resistance of arterial vessels, and it is also affected by aortic valve closure function. h3/h1 changes according to the changes of peripheral resistance and compliance of the vascular wall, and h4/h1 increases with the increase of peripheral resistance of the vascular wall. There are obvious differences among the signal characteristics of six pulse conditions. And the accuracy of fuzzy neural network in pulse recognition is significantly higher than that of BP neural network and fuzzy $\mathrm{C}$ mean $(\mathrm{P}<0.05)$.

In conclusion, the characteristic parameters of pulse signal are related to the arterial condition of human body, and the vascular condition and disease severity of patients can be inferred according to the specific values of characteristic parameter of pulse signal. Different pulse conditions can be distinguished according to the characteristic parameters of pulse signals. And fuzzy neural network can effectively improve the accuracy of pulse recognition and can be applied to clinical pulse diagnosis in the future.

\section{Acknowledgements}

Name of fund: Scientific research project of Yiwu Industrial \& Commercial College: Study on pulse signal recognition of TCM

Grant No.:ZD2020JD372-01

\section{References}

[1] Morozov V. A., Petrov Yu. V., Sukhov V. D. Experimental Evaluation of Structural and Temporal Characteristics of Material Fracture Based on Magnetic Pulse Loading of Ring Samples. Technical Physics, 2019, 64(5), pp. 642-646.

[2] Dadras E Y, Yazdani A, Nicknam A, et al. Incorporating Source Rupture Characteristics into the Nearault Pulse Prediction Model. Bulletin of the Seismological Society of America, 2018, 108(1), pp. 200-209.

[3] Su Q, Wang T, Ma W. Amplified characteristics and supercontinuum generation of square-wave pulse fiber laser. Optical Engineering, 2018, 57(2), pp. 1.

[4] Young J J., Friedman J S., Panov F, et al. Quantitative Signal Characteristics of Electrocorticogr-

[5] aphy and Stereoelectroencephalography: The Effect of Contact Depth. Journal of Clinical Neuroph-physiology, 2019, 3, pp. 1.

[6] Rumyantsev A Y, Petrenko M V. Theoretical calculations of resonant signals in the atomic-beam quantum frequency standard with laser pumping and detection. Journal of Physics Conference Series, 2018, 1038(1), pp. 012086.

[7] Rajaei A, Esfandiar Y, et al. Analysis of Quantum-Dot Size Effect on Dynamic and Modulation Characteristics of InAS/InP (311)B QD-Laser Under Large-Signal Modulation. Journal of Nanoelectronics \& Optoelectronics, 2017, 12(10), pp. 1154-1161.

[8] Chen Z, Wang G, Wang X. Physical mechanism and response characteristics of unsaturated optical stopping-based amorphous arsenic sulfide thin-film waveguides. IEEE Photonics.Journal, 2019, PP(99), pp. 1-1. 
[9] Boscolo S, Audo F, Finot C. Impact of initial pulse characteristics on the mitigation of self-phase modulation by sinusoidally time varying phase. Optical \& Quantum Electronics, 2018, 50(2), pp. 62.

[10] Kong F, Zhang S, Lin $\mathrm{H}$, et al. Effects of nanosecond pulse voltage parameters on characteristics of surface charge for epoxy resin. IEEE Transactions on Dielectrics and Electrical Insulation, 2018, 25(6), pp. 2058-2066.

[11] Du B X., Xue J S., Su J G. Effects of ambient temperature on electrical tree in epoxy resin under repetitive pulse voltage. IEEE Transactions on Dielectrics \& Electrical Insulation, 2017, 24(3), pp. 1527-1536. 